\title{
Long-term hearing performance and soft tissue outcomes of Baha@ Attract system in patients with bilateral congenital microtia in a single center
}

\author{
Ying Chen ${ }^{1}$, You-zhou Xie ${ }^{1}$, Liu-Jie Ren ${ }^{1}$, Na Gao ${ }^{1}$, Wen-xiu Gu${ }^{1}$, Chen-long $\mathrm{Li}^{1}$, \\ YAOYAO FU ${ }^{1}$, Jun-li Zhang ${ }^{1}$, and Tian-yu Zhang ${ }^{1}$ \\ ${ }^{1}$ Fudan University Eye Ear Nose and Throat Hospital
}

August 9, 2021

\begin{abstract}
Abstract: Background: Baha Attract System was designed as non-skin penetrating bone conduction implant to minimize skin complication. However, there were insufficient studies on its long-time acoustic outcome and safety in microtia patients with auricle reconstruction surgeries, who have thinner retroauricular subcutaneous layer and theoretically vulnerable skin at the implant side. Objectives: To analysis the long-term hearing performance and soft tissue complications of Baha@ Attract System in microtia population. Study Design \& setting: A prospective cohort study at single tertiary referral center. Patients: Twenty Mandarin-speaking patients with congenital bilateral microtia who were underwent Baha@ Attract implantation with or without auricle reconstruction surgeries simultaneously. Main Outcome Measure(s): Comparative analysis of audiological test results including hearing thresholds and speech recognition in quiet and in noise were pre- and post-operatively performed. subjective benefit as measured with the SSQ Scale. Soft tissue outcomes were monitored and documented. Results: The mean follow-up time was 36.11 \pm 3.30 months. Significant and stable improvements in hearing threshold and speech understanding in noise and quiet were demonstrated with aided condition. Subject evaluation revealed high level of patients' satisfaction with the amplification in all the subscales. Adverse events including skin necrosis increased after one year post-operatively. Soft tissue evolution including sin thinning, erythema, epidermis atrophy, soft tissue atrophy, skin necrosis was seen or once developed in most of the participants. However, prevalence of severe soft tissue complication was not higher to non-microtia population. Conclusions: Baha@ Attract System provides favorable and stable hearing improvements for microtia patients. Combining plastic surgeries with implantation will not increase the prevalence of soft tissue complication. However, long-term soft tissue problems should be highly aware of even after one year post implantation. Key words: Baha Attract, microtia, hearing, soft tissue, skin necrosis
\end{abstract}

\section{Long-term hearing performance and soft tissue outcomes of Baha@ ${ }^{@}$ Attract system in patients with bilateral congenital microtia in a single center}

\section{Abstract:}

Background: Baha Attract System was designed as non-skin penetrating bone conduction implant to minimize skin complication. However, there were insufficient studies on its long-time acoustic outcome and safety in microtia patients with auricle reconstruction surgeries, who have thinner retroauricular subcutaneous layer and theoretically vulnerable skin at the implant side.

Objectives: To analysis the long-term hearing performance and soft tissue complications of Baha ${ }^{\circledR}$ Attract System in microtia population.

Study Design \& setting: A prospective cohort study at single tertiary referral center. 
Patients: Twenty Mandarin-speaking patients with congenital bilateral microtia who were underwent Baha ${ }^{\circledR}$ Attract implantation with or without auricle reconstruction surgeries simultaneously.

Main Outcome Measure(s): Comparative analysis of audiological test results including hearing thresholds and speech recognition in quiet and in noise were pre- and post-operatively performed. subjective benefit as measured with the SSQ Scale. Soft tissue outcomes were monitored and documented.

Results : The mean follow-up time was $36.11 \pm 3.30$ months. Significant and stable improvements in hearing threshold and speech understanding in noise and quiet were demonstrated with aided condition. Subject evaluation revealed high level of patients' satisfaction with the amplification in all the subscales. Adverse events including skin necrosis increased after one year post-operatively. Soft tissue evolution including sin thinning, erythema, epidermis atrophy, soft tissue atrophy, skin necrosis was seen or once developed in most of the participants. However, prevalence of severe soft tissue complication was not higher to non-microtia population.

Conclusions: Baha ${ }^{\circledR}$ Attract System provides favorable and stable hearing improvements for microtia patients. Combining plastic surgeries with implantation will not increase the prevalence of soft tissue complication. However, long-term soft tissue problems should be highly aware of even after one year post implantation.

Key words: Baha Attract, microtia, hearing, soft tissue, skin necrosis

\section{Key points:}

1. Baha ${ }^{@}$ Attract System can provide effective and stable hearing amplification for microtia patients.

2. Concurrent surgeries of auricle reconstruction and Baha ${ }^{@}$ Attract implantation will not bring additional risk in terms of soft tissue complications.

3 .Adevese events increased with the duration of the implantation, even one year post surgery.

4. Benign skin evolution including skin thinning, erythema at the implant side is commonly seen in Baha ${ }^{@}$ Attract users and need to be aware of.

5. Implant is not necessarily removed when skin necrosis occurs in Baha ${ }^{@}$ Attract users.

\section{Introduction}

Microtia is a common congenital malformation which can causes conductive or mix hearing loss. Hearing rehabilitation by meatoplasty and tympanoplasty for patients with microtia and atresia remains a great challenge for otologists due to the complex anatomy and potential complications. Only patients with Jahrsdoerfer grading 6 or higher are suitable candidates for tympanoplasty(1).

Bone conduction implants (BCIs) can be considered as alternation for hearing restore for microtia(2). Bone Anchored Hearing Aid (BAHA), as a representative percutaneous BCI, has been widely implanted in microtia with atresia cases. Common complications regarding to skin reactions were reported at a high rate of up to $\sim 30 \%$ due to its percutaneous abutment(3). Non-skin penetrating BCIs were designed to avoid the disadvantages of the percutaneous type. Baha ${ }^{@}$ Attract System was the late-launched transcutaneous BCI in 2013. Several studies have proved the efficiency of this system and the advantage of less soft tissue complications(4). It has been reported the Baha ${ }^{\circledR}$ Attract System implantation could be performed simultaneously with microtia auricular reconstruction(5).

Unlike other hearing impairment population, patients with bilateral microtia ideally should get early audiological intervention during childhood to meet the demands of speech development. Meanwhile, microtia usually occurred with the concomitnta dysplasia of the mandibular bone and temporal bone, resulting to a low volume of mastoid and tympanum pneumatization(6). During the concurrent surgery of auricular reconstruction and hearing aids implantation when the candidate was six years or older, taking the mastoid volume for holding the implant into account, it's wisely to adopt a comparably small implant. To this extend, 
Baha ${ }^{@}$ Attract System is more suitable to be implanted in order to avoid possible compression of the sigmoid sinus and meninges during the implantation(6).

Moreover, the safety of skin needs to be identified by clinical observation, when the subcutaneous retroauricular fascial flap was rotated during the simultaneous surgery, leading to thinner subcutaneous layer and theoretically vulnerable skin at the implant side. This study reported long term soft tissue outcomes as with as hearing performance of Baha ${ }^{\circledR}$ Attract implant, aiming to provide solid evidence to the current knowledge.

\section{Method and material}

The current manuscript was prepared with STROBE checklist.

\subsection{Ethical standards}

This study was approved by the local ethical committee and was conducted in accordance with the ethical standards of the 1964 Declaration of Helsinki. Informed consent was obtained from all individual participants or their supervisors before recruitment.

\subsection{Study design}

Single-center prospective cohort study. Patients served as their own controls.

\subsection{Patience and device}

Bilateral microtia patients with an indication for a bone conduction hearing implant in this study were recruited from 2017/7/1-2018/3/1. All recipients should meet audiological eligibility. Test device was the Baha $^{@}$ Attract System (Cochlear Bone Anchored Solutions AB, Molnlycke, Sweden) with Baha 5 sound processor (SP). Test osseointegration implant was BI300 Implant on which BIM400 Implant Magnet was fixed. Strengths of SP magnets available for participants vary from the strongest (No.6) to the weakest (No.1) to provide sufficient retention.

\subsection{Surgery}

Implantation was performed solely or integrated with staged auricle reconstruction (framework projection) for microtia simultaneously under general anesthesia. During integrated surgeries, incision $5 \mathrm{~mm}$ outside the posterior margin of the reconstructed auricle was made first, superficial layer of the fascia covering the dorsal part of reconstructed auricle was dissected to the posterior edge of the cavum conchae, the retroauricular fascial flap $20 \mathrm{~mm}$ behind the incision was dissected and lifted to match the expected cranioauricular sulcus(7). With lifting of the retroauricular fascia, the periosteum at implant site could be sufficiently exposed, causing thin soft tissue layer above the implant. Then the BI300 was implanted in a standard surgical procedure, soft tissue reduction was performed if the flap thickness was more than $6 \mathrm{~mm}(8)$. Following procedure was conducted as previously reported(7).

Four weeks post-operation, if the wound had healed, the Baha SP was loaded though magnet coupling during first fitting. Afterwards, soft tissue over the implant was evaluated and documented by same senior surgeons.

\subsection{Audiometry and speech recognition test}

Audiometry was performed in a soundproof booth systematically at the fist fitting, 3-,6-months and 3-years after fitting. Hearing threshold was measured in free field test using warble tone. Speech recognition in quiet and in noise were measured using Mandarin Speech Test Material. To measure speech recognition in quiet, monosyllabic and disyllabic speech discrimination test at $65 \mathrm{~dB}$ SPL were performed(9). For speech recognition in noise, sentences consisted of 6-9 Chinese characters (6-9 syllables) and white noise at +5 or $+10 \mathrm{~dB}$ signal-to-noise ratio (SNR) were applied. All the test signals were presented to the front side of the participants. Measurements were repeated 2-4 times and average values were taken to avoid implementation bias.

\subsection{Subjective benefit}


The Speech, Spatial and Qualities of Hearing Scale (SSQ) questionnaire was administered pre-and postoperatively to evaluate the subject benefit in three subscales when using hearing amplification(10).

\subsection{Statistical analysis}

Results are presented as mean +- SD. Bone-conduction or air-conduction pure tone average (BC/AC-PTA) was the mean of hearing thresholds on frequency $0.5,1,2,4 \mathrm{kHz}$. The hearing gain was meant as unaided PTA minus aided PTA in the free field test. Statistical analysis was conducted utilizing SPSS (SPSS Inc., Chicago, USA, v22.0). P values of $<0.05$ were considered as statistically significant.

\section{Results}

\subsection{Demography}

Twenty Mandarin speaking patients (18 males and 2 females) were recruited, including two mix hearing loss patients and 18 cases with conductive hearing loss. Mean age during implantation was 14.67+-6.02, ranging from 8 to 30 years old. Clinical follow-up was completed in 18 patients (90\%) with a mean duration of 36.11+-3.30 months(31-44months), among which 16 cases underwent final audiological assessment. Two patients (10\%) were lost to follow-up 6 months post fitting.

\subsection{Baseline hearing}

Baseline audiometry revealed an average BC-PTA of $16.0+-7.21 \mathrm{~dB}$ HL of the implanted ears, ranging from $1.25-32.50 \mathrm{~dB} \mathrm{HL}$, and an average AC-PTA of $75.1+-12.63 \mathrm{~dB}$ HL. Baseline hearing thresholds in frequencies are showed in Figure 1.

\subsection{Surgery and wound healing}

Perioperative period was uneventful except that one girl (No.4 patient) was found hematoma 6 days postoperatively, and recovered after hematoma puncture and compression bandage. Integrated surgery was performed in 16 patients, the remaining 4 patients underwent implantation only, among them 2 cases completed auricle reconstruction surgeries previously. Sound Processors were firstly switched on as scheduled one month after surgeries in all participants.

\subsection{Skin thinness and magnet}

Soft tissue reduction was applied in one patient from $8 \mathrm{~mm}$ to $6 \mathrm{~mm}$. Mean skin thickness was $4.63+-1.14 \mathrm{~mm}$ at the implant sides. During the first half year, a trend of downgrading the magnets during follow-up was observed (Table 1). One patient suffered from insufficient retention force around 2 years after integrated surgery, which was solved by changing into stronger magnet. B-ultrasound examination showed scar tissue grew near his implant, resulting in different soft tissue thickness above the implant.

\subsection{Audiometry results}

Mean AC hearing thresholds in the free field test on each frequency by visit are shown in Figure 2A. AC hearing threshold averages with softband or implants improved significantly compared to the unaided condition in all frequencies (pi0.001). The unaided-AC PTA was $59.43+-4.54 \mathrm{~dB}$ SPL, which decrease to a aided PTA of 24.94+- $3.76 \mathrm{~dB}$ SPL(n=20), $25.31+-3.76 \mathrm{~dB}$ SPL(n=20)and 27.34+-9.28 dB SPL(n=16)at 3,6 months and 3 years, respectively. No statistical differences in PTA compared with each two results was recorded. Comparing PTA between unaided and aided hearing at 6 months, a hearing gain of $34.13+-4.97$ $\mathrm{dB}$ was achieved. A maximum hearing gain of $44.75+-5.73 \mathrm{~dB}$ was observed in the frequency of $1 \mathrm{kHz}$ (Figure 2B).

The result of the speech recognition score in quiet and noise is summarized in Figure 2C. The aided score showed significant improvements in all conditions compared with unaided hearing $(\mathrm{p}<0.001)$. Notably, monosyllabic and disyllabic WRS average when aided by implants (3 year) were significantly better than by softband $(\mathrm{p}<0.05)$.

\subsection{Subjective benefit}


Patient- or parents-reported SSQ questionnaires showed a significant improvement between unaided and aided conditions in speech $(\mathrm{p}<0.05)$, spatial $(\mathrm{p}<0.05)$, and quality $(\mathrm{p}<0.05)$ (Figure 2D). A gross score of 6.36 in 6 months post-fitting was achieved, which was significantly better than the pre-operative score of $4.44(\mathrm{p} ; 0.001)$.

\section{7 Adverse events and soft tissue outcomes}

Adverse events were definite as soft tissue or skin complication that made users stop using the devices, which increased from 4 cases at 6 months to 13 cases at 3 years (Table 2). Most of the adverse events were minimal and recovered without medical intervention.

However, two events were ranked as severe ones because of causing a break of more than one month. One severe adverse event (SAE)was reported about implant exposure and infection in No.4 patient 2 years after surgery (Figure 3A). Infection was rapidly controlled with conservative treatment (including appropriate antibiotic treatment, daily rinse with hydrogen peroxide and ofloxacin). The subcutaneous tissue but not the skin re-grew to cover the implant, so a surgical debridement and skin flap transposition was performed to cover the uneven surface 4 months later. Afterwards, it took another 2 months after reverse surgery for the patient to re-use the device with a gradually increasing duration, varied from 5 to 11 hours per day. Numbness occurred after long duration of device wearing in decreasing frequency and severity. Audiometry evaluation revealed that the implant system still yielded a good performance in aided condition.

The other SAE happened in No.21 patient who developed extreme soft tissue atrophy after wearing his magnet system without softpad. A Softband was applied for 1.5 months until the patient recovered (Figure 3B).

In the last re-visit, soft tissue and skin modification or complication was seen in $72.2 \%(13 / 18)$ patients included soft tissue thinning, erythema, epidermis atrophy, and local hair loss that did no need further treatment (Table 2, Figure 3C-H). During the whole study, 4 out of 18 patients experienced no skin irrigation, pain, numbness or other adverse events even these patients continuously used the devices.

\subsection{Duration of daily use}

At the end, all patients benefited from their devices. The girl recovered from implant exposure was still increasing the time of using the magnet system. The remaining patients reported regular use of the devices in all waken hours varied from 8 to 16 hours.

\section{Discussion}

\subsection{Synopsis of key findings}

The current study demonstrated the effective and stable hearing amplification of Baha ${ }^{@}$ Attract System, and make an alert by described high proportion of the long-term soft tissue evolutions in its microtia users. Meanwhile, clinical experiences of dealing with soft tissue problem including skin necrosis were provided for health care providers.

\subsection{Limitations and strengths}

This study was a single-center prospective trial. With longer follow-ups, the authors showed the unreported soft tissue evolutions and the corresponding treatment in the Baha ${ }^{@}$ Attract users, which is the crucial for the soft tissue safety. However, the sample size should be enlarged to increase the strengths of study.

\subsection{Compared to other studies}

\subsection{1 hearing outcomes}

Audiometry results confirmed that Baha ${ }^{@}$ Attract not only provided a satisfactory, stable hearing gains for microtia population, but also significantly improved speech recognition. The hearing test results consisted 
with that of other studies on microtia group, but were better than other studies including different etiologies $(5,11-13)$. As previously reported, downgrading magnet force without decreasing hearing gains was observed(14). The reason behind might be the gradually thinning of skin on the implants side.

\subsubsection{Soft tissue safety}

Previous studies on the Baha ${ }^{\circledR}$ Attract implantation combined with auricle reconstruction reported no adverse events $(5,20)$. However, most involved patients in these studies were followed up for no more than 6 months post-fitting. A study with one year follow-up reported skin thinning in Baha Attract users, but not fully elaborated the prognosis (14).

In the current study, eliminating two dropout cases, less than $1 / 3$ patients in our cohort were free of soft tissue evolution or complications. Moreover, the proportion of patients with adverse events increase from $20 \%(4 / 20)$ at 6 months to $55.6 \%(10 / 18)$ at $\sim 3$ years post-operatively, which was much higher than previously reviewed data(78.8\% users were free of skin complication) (15). Although most of the complications were minuscule or reversible, long term soft tissue complication should be aware of.

Another study by Nevoux reported high proportion of skin evolution in 20 Baha ${ }^{@}$ Attract users (putative non-microtia population with plastic surgeries) after following $2.9+-0.47$ years (16). Nevoux also proposed a three-grade classification of skin and soft tissue evolution for Baha ${ }^{@}$ Attract(16). According to this classification system, in our patients who underwent implantation and auricle reconstruction simultaneously or separately $(\mathrm{n}=18), 4$ cases (patients developed soft tissue atrophy, epidermis atrophy, hair loss respectively) could not be well classified, one patient went through grade 3 soft tissue complication, the rest 13 cases were ranked as grade 0-1 evolution that need no intervention. Fisher exact test provided there was no difference between these two cohorts in proportions of patients with grade $0-1$ skin reaction $(\mathrm{p} \dot{c} 0.05,75 \%$ cases in Nevoux's group ). Furthermore, there were more patients undergoing salvage surgeries in Nevoux's group (3 cases) compared to ours (1 case). The above facts demonstrated implantation combined with auricle reconstruction would not lead to higher prevalence of long-term complications.

\subsubsection{Clinical treatments for skin necrosis in Baha ${ }^{@}$ Attract users.}

Skin necrosis is the most severe complication leading to implant removal in Baha Attract users. There were only two case of skin necrosis previously reported by Chen and Nevoux, respectively(16, 17); In our skin necrosis case, conservative and surgical treatment were taken to maintain the implant. Many factors might play important role for the success of treatment. Firstly, the titanium implant ensured good biocompatibility(18); secondly, intensive care was administrated to control infection; thirdly, pedicled flap that exceeded beyond the boundary of implant magnet was made to guarantee its blood supplement; Finally, long break before re-loading the external magnet and slowly increasing the time of device wearing ensured the safety of the flap. Our experience provided another way to treat skin necrosis in Baha ${ }^{\circledR}$ Attract users, yet had apparent disadvantages of prolonged the unavailability of the magnet system. Medical decision should be made according to patients' preference, predicted clinical outcomes, health care resource and other socioeconomic factors.

\subsection{Clinical applicability}

The present study certified the long-term soft tissue safety of Baha ${ }^{@}$ Attract in microtia patient underwent auricle reconstruction and implantation simultaneously or separately, and proposed that more attention should be taken for the soft tissue evolution. Potential risk factors including overloading retention force, wearing magnet system without softpad, or long duration of device using without break should be avoided.

\section{Conclusion}

The investigated Baha ${ }^{@}$ Attract system benefits the bilateral microtia patients by providing favorable and stable hearing improvements. Integrated auricle reconstruction with implantation is safe in terms of soft tissue reaction. However, long-term soft tissue problems should be highly aware of even after one year post implantation. 


\section{Acknowledgement}

All the authors declared of no conflict of interest.

\section{References}

1. Jahrsdoerfer RA, Yeakley JW, Aguilar EA, Cole RR, Gray LC. Grading system for the selection of patients with congenital aural atresia. Am J Otol. 1992;13(1):6-12.

2. Ghossaini SN, Roehm PC. Osseointegrated Auditory Devices: Bone-Anchored Hearing Aid and PONTO. Otolaryngol Clin North Am. 2019;52(2):243-51.

3. Shapiro S, Ramadan J, Cassis A. BAHA Skin Complications in the Pediatric Population: Systematic Review With Meta-analysis. Otol Neurotol. 2018;39(7):865-73.

4. Gawecki W, Stieler OM, Balcerowiak A, Komar D, Gibasiewicz R, Karlik M, et al. Surgical, functional and audiological evaluation of new Baha((R)) Attract system implantations. Eur Arch Otorhinolaryngol. 2016;273(10):3123-30.

5. Fan X, Chen Y, Niu X, Wang Y, Fan Y, Chen X. Outcomes of the Baha Attract System combined with auricle reconstruction in mandarin speaking patients with bilateral microtia-atresia. Acta Otolaryngol. 2019;139(5):425-31.

6. Yang J, Zhao C, Liu Y, Gao M, Ren R, Wang D, et al. The effect of anatomical variables and use of the Lifts system on hearing outcomes after implantation of an active transcutaneous bone conduction device in bilateral congenital conductive hearing loss. J Otolaryngol Head Neck Surg. 2020;49(1):57.

7. Shan J, Guo Y, Chang KW, Zhang T. A modified technique for firm elevation of the reconstructed auricle. Eur Arch Otorhinolaryngol. 2016;273(10):3019-24.

8. Clamp PJ, Briggs RJ. The Cochlear Baha 4 Attract System - design concepts, surgical technique and early clinical results. Expert Rev Med Devices. 2015;12(3):223-30.

9. Wang S, Zhang H, Yue P, Wang L, Chen J, Ji C, et al. [Equivalence analysis of disyllabic Mandarin speech test materials]. Lin Chuang Er Bi Yan Hou Ke Za Zhi. 2006;20(13):590-3.

10. Noble W, Gatehouse S. Interaural asymmetry of hearing loss, Speech, Spatial and Qualities of Hearing Scale (SSQ) disabilities, and handicap. Int J Audiol. 2004;43(2):100-14.

11. Sylvester DC, Gardner R, Reilly PG, Rankin K, Raine CH. Audiologic and surgical outcomes of a novel, nonpercutaneous, bone conducting hearing implant. Otol Neurotol. 2013;34(5):922-6.

12. Briggs R, Van Hasselt A, Luntz M, Goycoolea M, Wigren S, Weber P, et al. Clinical performance of a new magnetic bone conduction hearing implant system: results from a prospective, multicenter, clinical investigation. Otol Neurotol. 2015;36(5):834-41.

13. Siegert R, Kanderske J. A new semi-implantable transcutaneous bone conduction device: clinical, surgical, and audiologic outcomes in patients with congenital ear canal atresia. Otol Neurotol. 2013;34(5):927-34.

14. Nevoux J, Coudert C, Boulet M, Czajka C, Tavernier L, Daval M, et al. Transcutaneous Baha Attract system: Long-term outcomes of the French multicenter study. Clin Otolaryngol. 2018;43(6):1553-9.

15. Dimitriadis PA, Farr MR, Allam A, Ray J. Three year experience with the cochlear BAHA attract implant: a systematic review of the literature. BMC Ear Nose Throat Disord. 2016;16:12.

16. Nevoux J, Boulet M, Nowak C, Le Pajolec C, Coudert C, Papon JF. Why skin is so important for Baha Attract success: A preliminary analysis of the first 20 implantations. Clin Otolaryngol. 2019;44(3):401-5.

17. Chen SY, Mancuso D, Lalwani AK. Skin Necrosis After Implantation With the BAHA Attract: A Case Report and Review of the Literature. Otol Neurotol. 2017;38(3):364-7. 
18. Iseri M, Orhan KS, Kara A, Durgut M, Ozturk M, Topdag M, et al. A new transcutaneous bone anchored hearing device - the Baha(R) Attract System: the first experience in Turkey. Kulak Burun Bogaz Ihtis Derg. 2014;24(2):59-64.

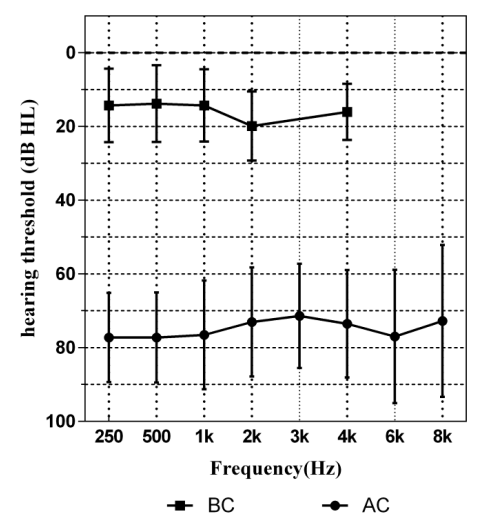

FIGURE.1 Mean AC-and BC- hearing level of the implanted ears(n=20,pre-op). Solid line with squares denotes mean $\mathrm{BC}$ thresholds, the line with dots means $\mathrm{AC}$ thresholds, and error bars show the standard deviation.

\section{Hosted file}

FIGURE 2.docx available at https://authorea.com/users/429810/articles/533427-long-termhearing-performance-and-soft-tissue-outcomes-of-baha-attract-system-in-patients-withbilateral-congenital-microtia-in-a-single-center 


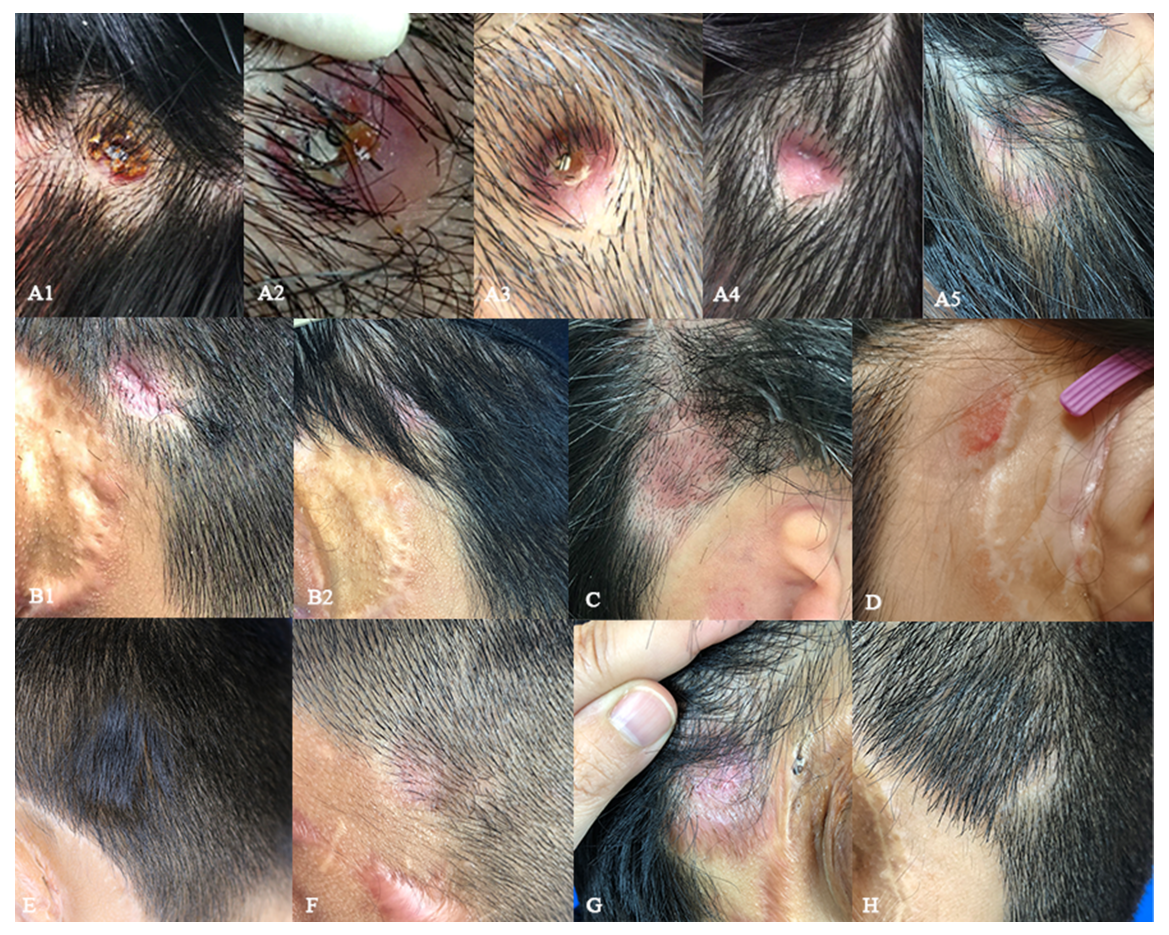

\section{Hosted file}

Tables 1.docx available at https://authorea.com/users/429810/articles/533427-long-termhearing-performance-and-soft-tissue-outcomes-of-baha-attract-system-in-patients-withbilateral-congenital-microtia-in-a-single-center

\section{Hosted file}

Tables 2.docx available at https://authorea.com/users/429810/articles/533427-long-termhearing-performance-and-soft-tissue-outcomes-of-baha-attract-system-in-patients-withbilateral-congenital-microtia-in-a-single-center 